\title{
Zurmühle, Jürg
}

\section{Musik im Kindergarten und in der Unterstufe der Primarschule}

Bachmann, Sara [Hrsg.]; Bertschy, Franziska [Hrsg.]; Künzli David, Christine [Hrsg.]; Leonhard, Tobias [Hrsg.]; Peyer, Ruth [Hrsg.]: Die Bildung der Generalistinnen und Generalisten. Perspektiven auf Fachlichkeit im Studium zur Lehrperson für Kindergarten und Primarschule. Festschrift für Frau Prof. Dr. Charlotte Müller. Bad Heilbrunn : Verlag Julius Klinkhardt 2021, S. 121-140. - (Studien zur Professionsforschung und Lehrerbildung)

Quellenangabe/ Reference:

Zurmühle, Jürg: Musik im Kindergarten und in der Unterstufe der Primarschule - In: Bachmann, Sara [Hrsg.]; Bertschy, Franziska [Hrsg.]; Künzli David, Christine [Hrsg.]; Leonhard, Tobias [Hrsg.]; Peyer, Ruth [Hrsg.]: Die Bildung der Generalistinnen und Generalisten. Perspektiven auf Fachlichkeit im Studium zur Lehrperson für Kindergarten und Primarschule. Festschrift für Frau Prof. Dr. Charlotte Müller. Bad Heilbrunn : Verlag Julius Klinkhardt 2021, S. 121-140 - URN:

urn:nbn:de:0111-pedocs-222084 - DOI: 10.25656/01:22208

https://nbn-resolving.org/urn:nbn:de:0111-pedocs-222084

https://doi.org/10.25656/01:22208

in Kooperation mit / in cooperation with:

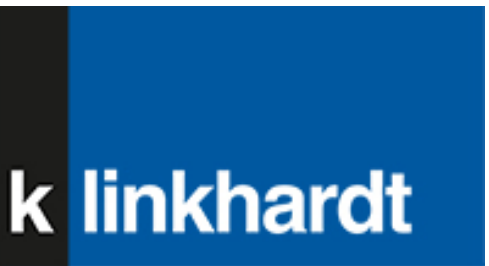

http://www.klinkhardt.de

\section{Nutzungsbedingungen}

Dieses Dokument steht unter folgender Creative Commons-Lizenz: http://creativecommons.org/licenses/by-nc-sa/4.0/deed.de - Sie dürfen das Werk bzw. den Inhalt unter folgenden Bedingungen vervielfältigen, verbreiten und öffentlich zugänglich machen sowie Abwandlungen und Bearbeitungen des Werkes bzw. Inhaltes anfertigen: Sie müssen den Namen des Autors/Rechteinhabers in der von ihm festgelegten Weise nennen. Dieses Werk bzw. der Inhalt darf nicht für kommerzielle Zwecke verwendet werden. Die neu entstandenen Werke bzw. Inhalte dürfen nur unter Verwendung von Lizenzbedingungen weitergegeben werden, die mit denen dieses Lizenzvertrages identisch oder vergleichbar sind.

Mit der Verwendung dieses Dokuments erkennen Sie die Nutzungsbedingungen an.

\section{Terms of use}

This document is published under following Creative Commons-License: http://creativecommons.org/licenses/by-nc-sa/4.0/deed.en - You may copy, distribute and transmit, adapt or exhibit the work in the public and alter, transform or change this work as long as you attribute the work in the manner specified by the author or licensor. You are not allowed to make commercial use of the work. If you alter, transform, or change this work in any way, you may distribute the resulting work only under this or a comparable license.

By using this particular document, you accept the above-stated conditions of use.

\section{Kontakt / Contact:}

\section{DeDOCS}

DIPF | Leibniz-Institut für Bildungsforschung und Bildungsinformation Informationszentrum (IZ) Bildung

E-Mail: pedocs@dipf.de

Internet: www.pedocs.de

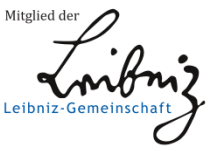


Studien zur Professionsfiorschung

und Lehrerbildung)

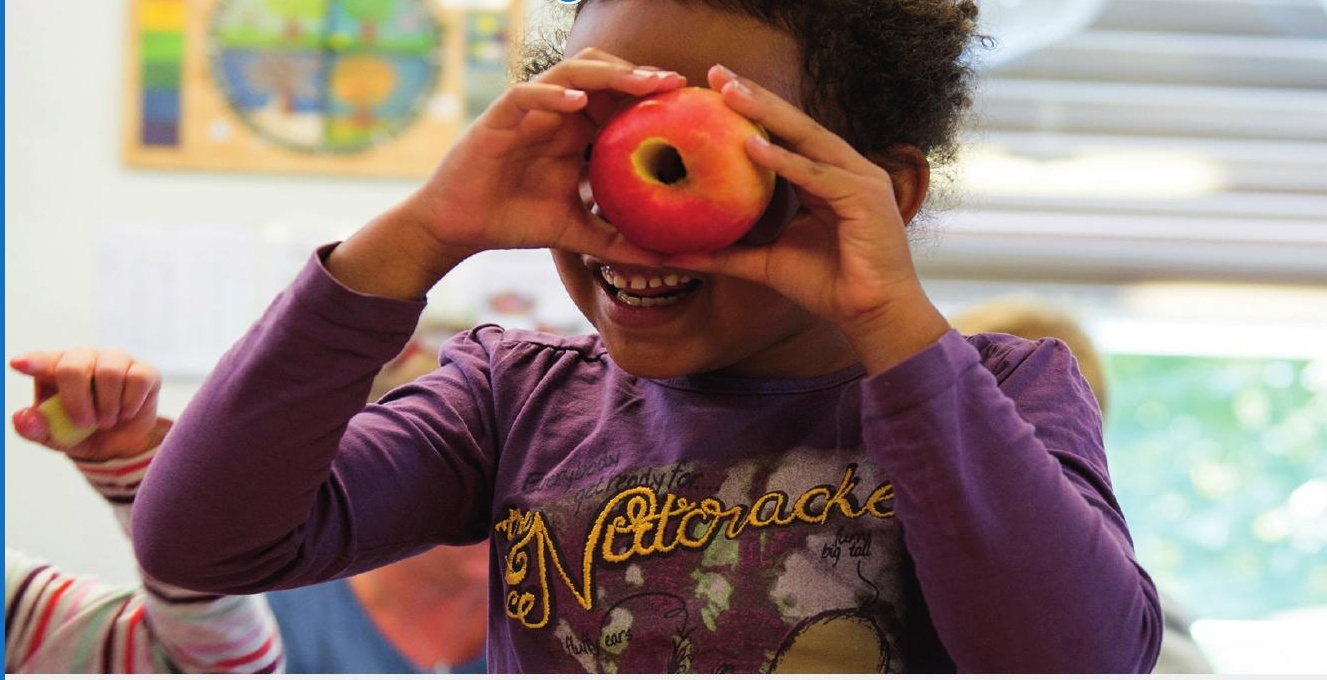

Sara Bachmann / Franziska Bertschy Christine Künzli David / Tobias Leonhard Ruth Peyer

(Hrsg.)

\section{Die Bildung der Generalistinnen und Generalisten}

Perspektiven auf Fachlichkeit im Studium zur Lehrperson für Kindergarten und Primarschule 
Bachmann / Bertschy / Künzli David / Leonhard / Peyer Die Bildung der Generalistinnen und Generalisten 


\section{Studien zur Professionsforschung und Lehrerbildung}

Herausgegeben von

Till-Sebastian Idel, Manuela Keller-Schneider, Katharina Kunze und Christian Reintjes 


\section{Sara Bachmann \\ Franziska Bertschy \\ Christine Künzli David \\ Tobias Leonhard \\ Ruth Peyer \\ (Hrsg.) \\ Die Bildung der Generalistinnen und \\ Generalisten}

Perspektiven auf Fachlichkeit im Studium zur Lehrperson für Kindergarten und Primarschule

Festschrift für Frau Prof. Dr. Charlotte Müller anlässlich ihrer Emeritierung als Leiterin des Instituts Kindergarten-/Unterstufe

Verlag Julius Klinkhardt 
Die Herausgabe des Bandes wurde von der Pädagogischen Hochschule FHNW finanziert.

Dieser Titel wurde in das Programm des Verlages mittels eines Peer-Review-Verfahrens aufgenommen. Für weitere Informationen siehe www.klinkhardt.de.

Bibliografische Information der Deutschen Nationalbibliothek Die Deutsche Nationalbibliothek verzeichnet diese Publikation in der Deutschen Nationalbibliografie; detaillierte bibliografische Daten sind im Internet abrufbar über http://dnb.d-nb.de.

2021.ig. () by Julius Klinkhardt.

Foto Umschlagseite 1: (c) Theo Gamper, Solothurn/Tobias Leonhard.

Druck und Bindung: AZ Druck und Datentechnik, Kempten.

Printed in Germany 2021.

Gedruckt auf chlorfrei gebleichtem alterungsbeständigem Papier. 


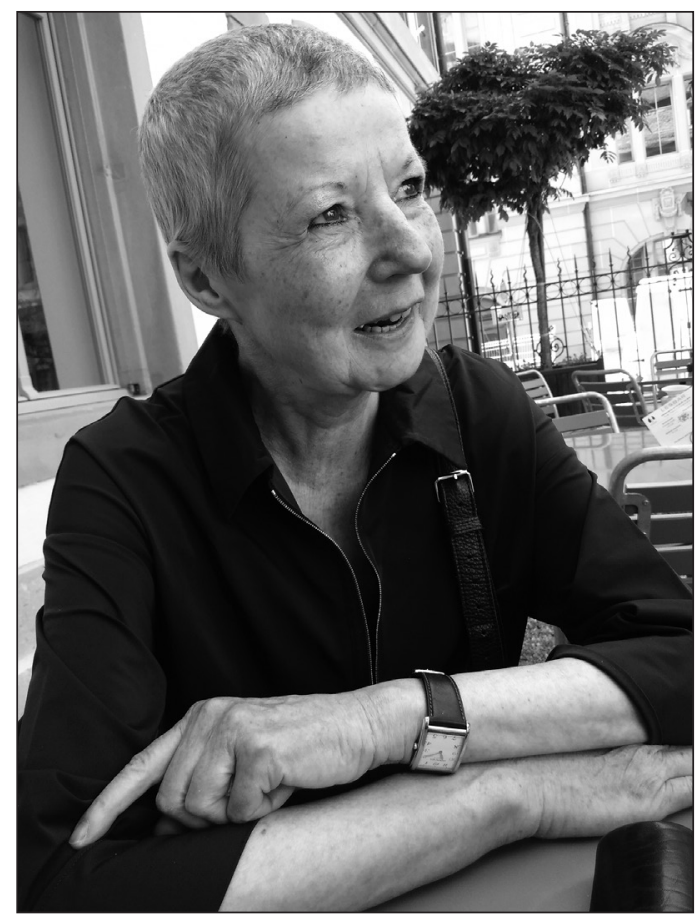

Prof. Dr. Charlotte Müller

Leiterin des Instituts Kindergarten-/Unterstufe

der Pädagogischen Hochschule FHNW von 2009 bis 2021 


\section{Inhaltsverzeichnis}

\section{Vorworte}

Sabina Larcher Klee

,Vielfachlichkeit und Eigensinniges

Sara Bachmann, Franziska Bertschy, Christine Künzli David, Tobias Leonhard und Ruth Peyer „Ich will jetzt endlich mal die Plots sehen!“

\section{Teil 1: Einführung und Problemaufriss}

Sara Bachmann, Franziska Bertschy, Christine Künzli David, Tobias Leonhard und Ruth Peyer

Die Bildung der Generalistinnen und Generalisten

Einleitung, Problemaufriss und Fragehorizont

Teil 2: Fachliche Perspektiven auf generalistische Handlungsanforderungen

Barbara Wyss

Flirten mit der Vernunft

Weltzugang und Welterzeugung durch Ästhetische Bildung

Bildnerisches und Technisches Gestalten im Zyklus 1

Elke Gramespacher, Susanne Störch Mehring,

Zita Bucher und Claudia Klostermann

Bewegungsbildung für Kinder:

Für „Generalistinnen“ und „Generalisten“ nicht nur eine sportdidaktische Herausforderung!

Mathilde Gyger

Zugänge zur Welt: sprachlich realisiert und didaktisch reflektiert Sprachliche Bildung im Zyklus 1 
8 Inhaltsverzeichnis

Christine Streit und Thomas Royar

Zahlen, Formen und Beziehungen

Lernen und Lehren von Mathematik im Zyklus 1

Jürg Zurmühle

Musik im Kindergarten und in der Unterstufe der Primarschule

Franziska Bertschy

Vielperspektivität und Perspektivenverbindung

Bildungsprozesse im Sachunterricht ermöglichen

Teil 3: Konzeptionen zur Stiftung einer professionsspezifischen Kultur von Fachlichkeit für den Zyklus 1

Christine Künzli David und Edwin J. de Sterke

Mehr als Fachlichkeit

Transversales Unterrichten als Spezifik einer Didaktik des Zyklus 1

und als verbindendes Konzept im Studiengang für Lehrpersonen

dieser Stufe

Tobias Leonhard

Fachlichkeit in zwei Praxen zur Geltung bringen

Beiträge der Berufspraktischen Studien zur fachlichen

Professionalisierung von Lehrpersonen des Zyklus 1

Anja Blechschmidt und Leticia Venâncio

Im Team für ALLE Kinder in der inklusiven Bildung unterwegs

\section{Nachwort}

Tobias Leonhard im Gespräch mit Charlotte Müller

Dem Eigensinn Raum geben

Annotationen zu Bildung in Kindergarten, Schule und Hochschule

Autorinnen- und Autorenverzeichnis 
Jürg Zurmühle

\section{Musik im Kindergarten und in der Unterstufe der Primarschule}

\section{Einleitung}

Kinder im Alter des ersten Zyklus erleben Musik auf vielfältige Weise. Musik ist als Singen, Bewegen und Tanzen, rhythmisches Sprechen, klangvolles Spielen mit Gegenständen und Instrumenten und als Hören selbstverständlicher Teil ihrer Lebensgestaltung. Im Kindergarten und in der Primarschule gehört Musik für alle Kinder als sinnliche und sinnvolle klingende Welt zum Alltag. Musik ermöglicht die Begegnung mit sich selbst als klangerzeugendes, klanggestaltendes und klangwahrnehmendes Wesen und rezeptiv und nachgestaltend als Begegnung mit der klingenden Welt aus unterschiedlichen Zeiten und Kulturen.

Vier reale Szenen aus dem Alltag von Kindergarten und Schule illustrieren nachfolgend unterschiedliche Zugänge von Kindern zur Welt der Musik und zur Welt durch Musik. Damit sind Fragen zum Gegenstand „Musik“, zur Bedeutung von Musik für die Kinder und zum Lernen und Lehren von und durch Musik in den schulischen Institutionen verbunden, die sich für die Ausbildung von Lehrpersonen in Kindergarten und Primarschule stellen.

Beispiel 1: Im Kindergarten singt Fatima der ganzen Kindergruppe ein Lied vor, welches sie als Türkin von klein auf kennt und welches mit einer für unsere Ohren ungewohnten Melodieführung und Rhythmik bezaubert. Die anderen Kinder und die Lehrperson lernen von ihr dieses Lied aus einer anderen Kultur in einer ihnen unbekannten Sprache und in einem fremden Duktus durch Zuhören, Imitieren und Wiederholung. Stichworte dazu: Musik aus anderen Kulturen, Lernen von- und miteinander durch Nachahmung und Wiederholung.

Beispiel 2: In der zweiten Klasse sitzen die Kinder vorne im Kreis und singen ein einfaches Lied, zu dem sie im Wechsel auf ihren eigenen und den Knien des neben ihnen sitzenden Kindern ein wiederkehrendes Muster patschen (Pattern). In ihrem musikalischen Spiel sind sie leiblich involviert und interagieren miteinander. Durch den Rhythmus und die Musik werden die Aktionen koordiniert, die Kinder begegnen sich selbst und anderen durch Bewegungen in sich wiederholenden zeitlichen Mustern. Sie hören der Musik aktiv zu und gestalten sie gleichzeitig mit. Die Musik ist Anlass zur koordinierten Bewegung und sozialer Interaktion und Kommunikation. Stichworte: Musik in Kommunikation und Koordination, leibliches Involviertsein. 
Beispiel 3: An einem Sommerabend präsentieren mehrere Klassen miteinander ein gesungenes und mit Instrumenten begleitetes Musical, zu welchem sie den Text und verschiedene Choreografien selbst erstellt haben. Hier wird Musik vorgeführt und ist Teil der kulturellen Aktivitäten der Schule und ermöglicht es den Kindern, sich mit ihrem Können anderen zu zeigen. Stichworte: eigene Gestaltung und Präsentation von musikalischen Handlungen, Teilhabe am kulturellen Leben, sich mit seinem musikalischen Können zeigen.

Beispiel 4: Raphael, im zweiten Jahr des Kindergartens, spielt zu Hause versonnen mit Legosteinen und singt Fragmente aus der Filmmusik aus „Starwars“ und „Pirates of the Caribbean“, aus dem alten Popsong „Popcorn“, aus dem Weihnachtslied "Jingle Bells" und aus vielen weiteren Liedern in einer längeren Phase ohne Unterbrechung vor sich hin. Er hat die Filme noch nie gesehen, das Stück „Popcorn“ nicht im Kindergarten gelernt. Er selbst weiss auf Nachfrage nicht, woher er die Musik kennt. Er hat sie aufgeschnappt und verwendet sie als Versatzstücke (vgl. Weber-Krüger 2014, 12-13) in seinem alltäglichen musikalischen Spiel. Musik ist Teil seiner Lebensgestaltung und seines persönlichen Ausdrucks auch ausserhalb des Kindergartens und ist Selbstzweck. Stichworte: Selbstbezug, unbeabsichtigtes Lernen, Selbstzweck der musikalischen Aktivität.

Die Beispiele und Stichworte deuten verschiedene Aspekte und Fragestellungen zum Musiklernen und -lehren an.

- Welche Musik wird zum Gegenstand des Unterrichts? Von welchen Begriffen von Musik wird ausgegangen? Musik aus welcher Kultur, aus welcher Zeit, aus welchem Stil wird thematisiert? Wird Musik als bestehendes Produkt und Werk oder Musik als Prozess und einmalige Improvisation verstanden? Knapp formuliert geht es um die Frage nach dem Gegenstand: Was ist Musik? (Abschnitt 2)

- Was wird den Kindern durch die Auseinandersetzung mit Musik und Musikmachen ermöglicht? Was lässt sich mit, durch oder über Musik erfahren? Welche Zugänge zur Welt und zu sich selbst sind spezifisch musikbezogen? Oder: Welche Bedeutung hat Musik im 1. Zyklus für die Kinder? (Abschnitt 3)

- Wie kann Lernen von Musik und durch Musik gefördert werden? Was kann durch, mit und über Musik gelernt werden? (Abschnitt 4)

- Was können Lehrpersonen in Kindergarten und Schule beitragen? (Abschnitt 5)

- Wie kann Musik im Rahmen der Ausbildung von Generalistinnen und Generalisten aufgebaut sein? (Abschnitt 6)

- Was können Musik und die musikalische Ausbildung zur generalistischen Ausbildung von Lehrpersonen beitragen? (Abschnitt 7)

Die Fragen sind nicht eindeutig und einfach zu beantworten, bleiben in der Diskussion und führen zu einer Pluralität einerseits von Musikdefinitionen (vgl. Altenmüller 2018; Dartsch/Knigge/Niessen/Platz/Stöger 2018; Geuen 2018) und 
andererseits von Unterrichtskonzeptionen (vgl. Lehmann-Wermser 2016; Ott 2018), auf die im Folgenden eingegangen wird.

Vorweg sind zwei Hinweise auf institutionelle Besonderheiten für das Verständnis des Kontexts der musikalischen Ausbildung und der musikalischen Tätigkeiten in Kindergarten und Primarschule notwendig:

1. Im Kindergarten wird der Musikunterricht in der Regel durch die Kindergartenlehrperson erteilt und ist integriert. In der Primarschule gibt es unterschiedliche Aufteilungen zwischen allgemeinem und in den Alltag in integrierten Unterricht durch die Klassenlehrperson und Unterricht in Halbklassen oder Kleingruppen in speziellen Zeitgefässen mit einer Fachlehrperson. ${ }^{1}$

2. Die musikalische Bildung ist auch Aufgabe der Musikschulen mit ihren Angeboten wie Eltern-Kind-Rhythmik ab zwei Jahren, musikalischer Früherziehung ab drei Jahren bis zum individuellen Instrumentalunterricht und Konzertbesuchen. Dieser Unterricht findet in der Regel an den Musikschulen statt und ist eine private Angelegenheit der Eltern und Kinder. Kooperationen zwischen der Institutionen Kindergarten/Primarschulen und $\mathrm{Mu}-$ sikschulen finden vermehrt und sehr erfolgreich statt. Diese Möglichkeiten werden durch Angebote von Kulturvermittlung, Orchestern, Theatern und weiteren Kulturschaffenden ergänzt. So sind die musikalische Bildung und die Ausbildung in den Händen verschiedener Akteurinnen und Akteure.

\section{Was ist Musik?}

Musik zu definieren und ihr Wesen zu fassen kann an dieser Stelle nur mit einigen Schlaglichtern angedeutet werden. Fragen, die sich stellen: Welche Musik aus welcher geografischen, zeitlichen und sozialen Kultur oder Subkultur ist Referenz für die Beschreibung von Musik? Geht es um die Reproduktion von bestehender oder um die Produktion von eigener Musik? Ist Musik ein Werk, ein Objekt oder eine Aktivität? Was ist Musik in der Wahrnehmung der Kinder? Was macht ein Rasseln mit selbst gebauten Instrumenten zur Musik? Sollen Bildung und Erziehung mit Musik oder zur Musik Ziele sein? Ist Musikmachen ein Selbstzweck oder ein Mittel zu etwas anderem? Welche theoretischen Grundlagen braucht es für einen verständigen Umgang mit Musik? „Die Antworten auf diese Fragen sind Legi-

1 In der Nordwestschweiz gibt es vier unterschiedliche Modelle: Kanton AG: Musikgrundschule mit einer Lektion pro Woche in der 1. und 2. Klasse; Kanton BL: musikalischer Grundkurs mit einer Lektion pro Woche in der 1. und 2. Klasse; Kanton BS: Musik und Bewegung mit einer Lektion pro Woche in der 1.-6. Klasse; Kanton SO: kein expliziter Fachunterricht in der Stundentafel ausgewiesen. 
on" (Schatt 2007, 32). Die hier ausgewählten und vorgestellten unterschiedlichen Perspektiven auf Musik, welche die Fragen umkreisen, haben Auswirkungen auf und Bedeutung für unterschiedliche musikpädagogische Konzepte, die für die Zielstufe vorliegen. Weggelassen werden in diesen Ausführungen Definitionen und Konzepte von Musik, die vorwiegend im Kontext von Musik als Kunstform entwickelt und diskutiert werden.

In einer dichten Beschreibung definiert Altenmüller Musik als Gegenstand, Produkt, Werk und Phänomen: „Musik sind bewusst gestaltete, in der Zeit gegliederte und nichtsprachliche akustische Ereignisse in sozialen Zusammenhängen“" (Altenmüller 2018, 9). Musik wird hier als Objekt beschrieben. Musik ereignet sich als klangliches Phänomen strukturiert in der Zeit, ist von Menschen bewusst gestaltet und enthält ihre Bedeutung in sozialen kulturell geprägten Kontexten.

Musik wird in einer anthropologischen Dimension und in ihrer Bedeutung für viele Menschen als ein „Lebensmittel“ aufgefasst: „Was wir Musik nennen, als Musik erleben, betreiben und verstehen und in unser Leben - es deutend und ordnend - einfügen, ist ein Stück und eine Möglichkeit unserer Lebensgestaltung" (Richter 2019, 16). In dieser Beschreibung geht es um subjektbezogene musikalische Erlebnisse, um eigene Handlungen und um ein Verständnis von etwas, was in das eigene Leben durch Deutung und Bedeutung strukturierend eingefügt wird. Der verstehende Umgang mit Musik benötigt Begriffe, Symbolisierungen und Theorien, die auf Erleben gründen und davon abstrahiert werden können.

Mehr auf die Wirkungen von Musik ausgerichtet beschreibt Dartsch: „Menschen modulieren sich und andere mittels Musik, sie definieren sich über Musik, sie kommunizieren und synchronisieren sich mit Musik, sie spielen mit Musik und sie bilden sich an ihr" (Dartsch 2010, 195). Die Wirkung von Musik kann zum Beispiel Stimmungen von Menschen verändern, trägt zur Bestimmung von sich selbst in Form eines musikbezogenen Selbstkonzepts bei (vgl. Spychiger 2013), ermöglicht Formen von nonverbalen Kommunikationen und Synchronisation im gemeinsamen Singen und Musizieren, ist eine Form des Spiels und ist Mittel, um sich in Wahrnehmung, Ausdruck und Verstehen zu bilden.

Musik kann, diesen nicht abschliessenden Bestimmungen folgend, als Werk, als Erlebnis, als Handlung, als Form einer Lebensgestaltung, als Bildungsinhalt, als Trägerin von bestimmten Bedeutungen und Wirkungen, als Möglichkeit der Selbstdefinition, als Medium der Kommunikation und als Spiel verstanden werden (vgl. Geuen 2018, 18). Diese Umkreisungen des Begriffs Musik lassen erahnen, dass der Umgang mit ihr in der Ausbildung von Lehrpersonen aus verschiedenen Perspektiven erfolgen muss. Musikunterricht in Kindergarten und Schule ist nicht auf das Singen von Liedern, auf das Lernen von musiktheoretischen Grundlagen wie Notenlesen oder auf musikalische Spiele zu beschränken. Zugleich muss dabei jedoch im Blick bleiben, dass ein Zuviel der Möglichkeiten 
nicht zu Verwirrung oder Erstarrung oder zu einer Haltung ,anything goes“ führt (Khittl 2017). Das gelingt durch bewusste Entscheidungen in Bezug darauf, was mit welcher Musik mit welchen Handlungen und welchen Begriffen welche Art des musikalischen Lernens ermöglicht.

Im Folgenden wird auf die Bedeutung von Musik (Abschnitt 3) und Musiklernen (Abschnitt 4) im Kindesalter eingegangen. Danach wird in Abschnitt 5 eine kleine Auswahl von unterschiedlichen musikpädagogischen Konzeptionen, die mit den unterschiedlichen Musikdefinitionen und Bedeutungen korrespondieren, skizziert und ein übergeordnetes Rahmenmodell vorgestellt, in welchem unterschiedliche Musikauffassungen und musikpädagogische Konzeptionen verortet werden.

\section{Bedeutungen von Musik für die Kinder im 1. Zyklus}

Die klangliche Welt der Kinder im Alter des Kindergartens und der Primarschule ist äusserst vielfältig. Musik ist in medialer Form fast permanent allgegenwärtig durch Kopfhörer in allen Ohren, als Untermalung in allen Filmen von YouTube bis zu „Blockbusters“. Poppige und rockige Kinderkonzerte sind beliebt und sehr gut besucht. Bewegen und Tanzen zur Musik, Imitieren von musikalischen Vorbildern, freies Spielen mit der Stimme und mit Instrumenten sind musikalische Aktivitäten seit Kleinkindheit an. Kinder sind informiert über ihre Lieblingsmusik und ihre Interpretinnen und Interpreten (Küntzel 2009, 6-7). Die Kinder kommen mit reichen und unterschiedlichen Erfahrungen, welche sie im nicht institutionalisierten und nicht formalen Umgang mit Musik gemacht haben, in den Kindergarten.

Schon im Kleinkinderalter werden Klänge und Bewegungen zwischen Kindern und Bezugspersonen koordiniert und synchronisiert. Im Kindergartenalter sind rhythmische Sprech-, Sing und Klatschspiele zentrale Formen von nonverbaler leiblicher Kommunikation, Koordination und Resonanz. Ohne die im Kleinkind angelegten und sich in der Entwicklung immer mehr ausdifferenzierenden Koordinations- und Resonanzfähigkeiten als grundlegende musikalische Kommunikationsformen, welche jede musikalische Aktivität durchdringen, sind Musikerleben und Musikmachen und mimetisches Lernen durch Vormachen und Nachmachen nicht möglich. Musikalische Spiele als durch Regeln strukturierte Formen des entdeckenden, aktiven Handelns mit Stimme, Bewegung und Instrumenten in einer Gruppe sind alltägliche Aktivitätsformen, die Spass machen: „Musik ist ihrem Wesen nach [...] eine besondere Form des Spiels“ und „Spiel und Experiment sind evolutionär bewährte Wege für den Erwerb von Grunderfahrungen“ (Dartsch 2010, 250-251). Auch Instrumente werden, gespielt . 
Als Aufforderung und normative Setzung betitelte Wilfried Gruhn 2003 ein Buch mit „Kinder brauchen Musik!“ (Gruhn 2003) und begründete dies wie folgt: „[S]ie brauchen Töne, die klingen und sich rhythmisch bewegen. Sie brauchen es für die Entwicklung ihrer Sinne, für ihr kreatürliches Wohlbefinden, für ihre allmähliche Erfahrung von Raum und Zeit und für die Vorstellung von musikalischen Strukturen, die im Bewusstsein verankert werden" (Gruhn 2003, 11). In der doppelten Bedeutung von „brauchen“ verstanden, benötigen und benutzen Kinder Klänge und Rhythmen schon seit ihrer frühesten Kindheit. Schon im Uterus hört das Kind Klänge, welche es nach seiner Geburt wiedererkennen kann (Podbregar 2013). Als Kleinkind nimmt es die Stimme der nahen Bezugspersonen wahr, welche sich in verschiedenen Lauten äussern, und es hört die vielen Klänge in seiner Umgebung. Singen wird mit Schaukeln und Wiegen mit rhythmischen Bewegungen verbunden. Das Kind selbst äussert sich mit Lauten und geräuschhaften Bewegungen, wie zum Beispiel mit sich wiederholendem Schütteln von Rasseln oder Klopfen mit Gegenständen. Diese elementaren sensomotorischen Aktivitäten (Schallwahrnehmung, Vokalisation, Bewegung) differenzieren sich zunehmend aus und passen sich allmählich an die Umgebung und Kontexte an (Stadler Elmer 2015, 9).

Musik ist Medium des Selbstausdrucks mit der eigenen Stimme, mit dem eigenen Körper und mit Instrumenten im weitesten Sinne (lat. ,instrumentum': ,Werkzeug') und eröffnet schon früh die Möglichkeit der Beeinflussung und der Modulierung der eigenen Befindlichkeit durch Klänge und erhöht dadurch das „Wohlbefinden“. Vom ersten Schrei nach der Geburt über die Brabbel-, Summund Kickserlaute differenziert das Kind seine klanglichen Äusserungen aus, koordiniert seine Stimme, seine Atmung und seine Bewegungen durch Feedback mit Klängen. Sprechen und Singen sind im frühen Kindesalter kaum unterscheidbar, sie „verschmelzen“ (Stadler Elmer 2015, 8). Musik ermöglicht einen sinnlichen, leibbezogenen, im engeren Sinne einen ästhetischen (von ,aisthesis': „sinnliche Wahrnehmung/Erkenntnis') Zugang zur Welt, zu sich selbst und zu anderen (vgl. Böhme 2001; Brandstätter 2013; Dartsch 2014, 64).

Schon früh haben Kinder „sensomotorischen Zugang zu den musikalischen Spielregeln in der es umgebenden Kultur. Diese Spielregeln sind ein Teil der kulturellen und rituellen Praxis und werden sozial erfahren in Form von gegenseitiger Nachahmung und Angleichung" (Stadler Elmer 2015, 178). In folgenden Phasen werden diese implizit erfahrenen Regeln verallgemeinert und angewendet, es wird handelnd klar, wie ein Lied zu klingen hat, damit es als Musik in einer bestimmten Kultur gilt. Später werden die Regeln bewusster genutzt und andere musikalische Phänomene in Begriffe gefasst. Musikalische Grundbegriffe wie Ton, Klang, Tonhöhe, Dauer, Puls, Takt, Akkord oder Intervall und Strukturen in der Musik wie Tonsysteme, Qualitäten von Zusammenklängen, Liedstrukturen oder rhythmische Hierarchien können erfasst und verstanden werden. Musikalische 
Tonhöhenbeziehungen und rhythmische Verhältnisse werden durch Übertragung in Körperbewegung und Sprechsilben in der Solmisation deutlich gemacht. Unterschiedliche Formen von Notationen ermöglichen das Festhalten und Neugestalten der flüchtigen Musik und das Reproduzieren von tradierten Stücken.

Begegnungen mit Musik aus verschiedenen geografischen, historischen und sozialen Kulturkreisen eröffnen Kindern durch Hören oder Selbstmusizieren Zugänge zur Welt der Musik und zur Musik der Welt, wie Beispiel 1 zu Beginn des Beitrags in Abschnitt 1 aufzeigt. Kinder zeigen gegenüber ungewohnter Musik in jüngeren Jahren eine höhere Bereitschaft, sich damit zu beschäftigen. „[M] usikalische Präferenzen [...] und musikalischer Geschmack [...] [sind] in den ersten zehn Lebensjahren noch nicht so ausgeprägt und daher weniger festgelegt [...] wie in späteren Lebensjahren“ (Gembris 2013, 331).

\section{Lernen von Musik}

Im Kindergarten beginnt für viele Kinder ein institutionalisiertes und strukturiertes Lernen mit und von Musik. Eine musikalische Grundbildung wird angestrebt, die auf „Förderung von Kreativität, performative Fertigkeiten und ästhetischen Sinn sowie auf die Vermittlung von Kenntnissen in Kunst und Kultur" ausgerichtet ist (D-EDK 2016, 2). Durch unterschiedliche Aktionsweisen wie Sensibilisierung, Exploration und Improvisation, die Gestaltung in verschiedenen Inhaltsbereichen wie Singen, Instrumentalspiel, Bewegung, Wahrnehmen und Erleben, Denken und Symbolisieren sowie das Verbinden von Musik mit anderen Gestaltungsformen werden die Kinder in die Grundlagen der Musik eingeführt (Dartsch 2014, 88-96; D-EDK 2016). Sensibilisierung meint Differenzierungen des sinnlichen Erspürens und Wahrnehmens im Hören, in der Bewegung des eigenen Körpers und des Tastens und Greifens. Es braucht dazu anregende Situationen, Materialien und soziale Rahmungen. Exploration, Improvisation und musikalische Gestaltungen sind Formen des schöpferischen klanglichen Ausdrucks des Kindes auf spielerische Weise. Dabei kann das Experimentieren mit Klängen von Impulsen der Lehrperson oder von den Kindern selbst ausgehen. Kinder werden zu „Klangforschenden und Musikgestaltenden“, indem sie in das Sinnesereignis ,Musik ' eintauchen, in klangerzeugenden Bewegungen aufgehen, mit Klängen Vorstellungswelten und Bedeutungen erkunden und selbst Urheber*innen von Musik sind (vgl. Beck-Neckermann 2014). Als elementare grundlegende Orientierungs- und Strukturierungserfahrungen werden Klänge im Tonraum in diskrete, voneinander abtrennbare Tonhöhen und in der Zeit durch Dauern ausdifferenziert. Die musikalischen Strukturen wie die Unterscheidungen von Konsonanz und Dissonanz, Gleichzeitigkeit und Abfolge, die verschiedenen 
Ton- und Taktarten, musikalische Formen mit Wiederholungen und Variationen werden handelnd durch Imitation und Improvisation erfahren und erst allmählich begrifflich erfasst und symbolisiert (vgl. Dartsch 2014; Gruhn 2010; Stadler Elmer 2015).

Musik ist auch ein Medium, mit dem etwas gestaltet und gelernt wird. Musik im Alltag von Kindergarten und Schule dient beispielsweise zur zeitlichen Strukturierung mit thematisch passenden Liedern und Musikspielen (Tagesstruktur, Wochenstruktur, Jahresstruktur), als Unterstützung und Begleitung von sozialen Ritualen und als nonverbales Kommunikationsmittel (Signale, Zeichen). Rhythmen und Strukturen sind im Zusammenhang mit Sprache, Mathematik, Bewegung und Bild klanglich und körperlich erfahrbar. Lieder erleichtern das Lernen von Texten, ein kleinstes gemeinsames Vielfaches kann getanzt werden, Bilder werden vertont oder Klänge in Bilder umgesetzt. Musik bietet Möglichkeiten zum nicht sprachlichen, metaphorischen sinnlichen Erkennen (vgl. Brandstätter 2013).

Musiklernen wird mit Begriffen wie ,musikalischer Begabung', ,musikalischem Talent' oder ,Musikalität' (ein Kind ist musikalisch oder nicht musikalisch) in Verbindung gebracht. Damit werden uneinheitlich definierte Eigenschaften der Kinder in Bezug auf Musiklernen umschrieben, die sie mitbringen oder erworben haben. Diese Eigenschaften erleichtern den Kindern den sinnhaften Umgang mit und das Lernen von Musik und werden durch genetische, soziale und motivationale Faktoren beeinflusst (Dartsch 2014,11-50; Gembris 2013, 23-30). Alle gesunden Kinder haben musikalische Fähigkeiten von klein auf. Sie können hören und das Gehörte unterscheiden, sie können singend und sprechend vokalisieren, sie können sich zum Erzeugen von Klängen und zu Klängen bewegen, sie sind motiviert, spielend und versunken musikalisch aktiv zu sein, sie können mimisch, gestisch, lautmässig imitieren und sie können kommunizieren (Stadler Elmer 2014).

Trotz der oben ausgeführten Vielfalt der Gegenstandsbestimmung und der Vielzahl von musikpädagogischen Konzeptionen können thesenhaft einige Grundprinzipien benannt werden, die für das Verständnis und die Ermöglichung von Musiklernen im Zyklus 1 leitend sind. Diese werden hier zusammenfassend und vereinfachend dargestellt.

\section{Gemeinsam handelnd in Bewegung in Phasen durch Wiederholen und Üben}

Wie in Abschnitt 1 erwähnt, findet Musikunterricht in Kindergarten und Schule im Gegensatz zur Musikschule ausschliesslich in Gruppen statt. Die Kinder sind gleichzeitig in Interaktion und Kommunikation, haben gleichwertige Rollen und sind individuell verantwortlich und gegenseitig voneinander abhängig (Evelein $2015,16)$. Imitierendes und mimetisches Lernen basieren grundlegend und von klein auf auf nonverbaler Kommunikation und Interaktion (Stadler Elmer 2015, 117). 
Musiklernen realisiert sich durch Handlung und den bewegten Körper (Fuchs 2010; Gruhn/Röbke 2018; Küntzel 2009). Einen Puls zu spüren und nachzuvollziehen, einen gemeinsamen gleichen Ton singen zu können, den Verlauf einer Melodie zu spüren und diesen singen zu können, Spannungen zwischen Dissonanz und Konsonanz zu erfahren oder Klänge mit Stimme oder Instrumenten zu erzeugen ist immer verbunden mit körperlichen oder leiblichen Aktivitäten und Erfahrungen. Klang ist seinem Wesen nach Bewegung, die durch Bewegung erzeugt und wahrgenommen wird. Die fundamentale Unterscheidungsfähigkeit zwischen "gleich“ und „verschieden" bei Klängen ist ein leiblich erfahrbarer Abgleich zwischen dem Gehörten und dem Selbstklingenden. Zwischen den Fähigkeiten der Bewegungskoordination und des Bewegungsflusses von Vorschulkindern bestehen positive Korrelationen mit deren melodischen und rhythmischen Fähigkeiten (Gruhn 2017, 109).

Die Beschreibung von Musiklernen in Phasen oder Stufen folgt der musikbezogenen Lerntheorie von Edwin Gordon und deren Weiterentwicklung durch Wilfried Gruhn (Gruhn 2010, 2014, 2017; Gruhn/Röbke 2018). Die durch Imitation und Exploration angeregten musikalischen Äusserungen der Kinder führen durch Wiederholungen zu erinnerbaren und wiederholbaren Mustern, denen neuronale Repräsentationen entsprechen. Kleine melodische Phrasen in einer bestimmten Abfolge oder rhythmische Pattern werden nachgemacht und können erinnert und wiedergegeben werden. Diese werden in einem zweiten Schritt benannt und strukturiert. Tonhöhenbeziehungen können durch relative Solmisationssilben bezeichnet werden, rhythmische Muster durch Rhythmussprachen. Erst dann werden Symbole und Notationen verwendet und die Theorie zu Musik eingeführt. Kinder können Dreiklänge oder Intervalle korrekt singen oder Synkopen korrekt klatschen, bevor sie diese Phänomene benennen oder ab Noten lernen können.

Die erworbenen Fähigkeiten werden durch Wiederholungen und Üben erweitert und gefestigt. Dafür braucht es Zeit, da, wie oben erwähnt, musikalisches Lernen vorwiegend über wiederholte Handlungen und leibbezogene Erfahrungen geschieht. Es reicht nicht, zu verstehen, wie etwas gesungen wird, sondern es muss zuerst ausgeübt und gefestigt werden können. Sinnvolles Üben erfordert die Möglichkeit, sich darauf einzulassen; die Achtsamkeit ist ausgerichtet auf das, was geübt oder wiederholt werden muss.

\section{Lehren von Musik}

Die Zielsetzung der Ausbildung ist, Studierende zu befähigen, von ihren eigenen musikalischen Fähigkeiten ausgehend innerhalb des zeitlichen und strukturellen 
Rahmens Musik als Fach und ästhetische Praxis gemeinsam mit den Kindern vielfältig und kompetenzorientiert unterrichten zu können. In der Ausbildung zur Lehrperson Kindergarten/Unterstufe müssen alle Studierenden das Fach ,Musik wählen. Die Vorerfahrungen mit Musik und musikalischer Ausbildung und die Fähigkeiten und Kenntnisse der Studierenden sind sehr unterschiedlich. Häufig besteht ein grosser Respekt vor dem Fach und eine niedrige Selbsteinschätzung. In Studien in Deutschland und in angloamerikanischen Ländern zeigte sich, dass die Einschätzung der Fähigkeiten, die es braucht, um Musik zu unterrichten, häufig um unteren Ende der Skalen zu finden sind (Hammel 2011; Schellberg 2016, 143-145). Diese Einschätzung hat, so ist zu vermuten, viel mit den tradierten und engeren Vorstellungen von Musik und Musikunterricht zu tun, die, so ist zu hoffen, durch die Auseinandersetzungen mit einem offeneren Verständnis von Musik und Musikunterricht und den damit gemachten Erfahrungen während der Ausbildung irritiert und erweitert werden können.

Die Lehrperson muss mindestens das können und wissen, was sie den Kindern vormacht, damit diese es richtig durch Imitation gemeinsam lernen können. Das ist selbstverständlich im Anspruch, jedoch weniger selbstverständlich anzutreffen in der Realität. Die Lehrpersonen müssen Lieder melodisch und rhythmisch korrekt und ansprechend singen, Bewegungen und Tänze zu Musik mit Energie und Freude ausführen, ihre eigene Wahrnehmung differenzieren und beschreiben, mit anderen zusammen musizieren, selbst Musik erfinden und notieren und die musiktheoretischen Grundlagen und Musik im Kontext der Kultur verstehen. Das sind fachliche Bereiche, die in der fachwissenschaftlichen Ausbildung ${ }^{2}$ im Vordergrund stehen. Die Studierenden entwickeln ihre schon unterschiedlich vorhandenen Fähigkeiten in der Ausbildung unterschiedlich. Es soll möglich sein, guten Unterricht auf sehr verschiedene Weise zu gestalten. „Da die Qualität von Unterricht vorrangig von der Lehrerpersönlichkeit abhängt, gibt es also keinen vorbildlichen Unterricht als Konzept. [Es geht] um Anstösse, um Ideen [...], mit denen jede Lehrerpersönlichkeit auf ihre ureigene Weise verfährt" (Küntzel 2009, 75).

Um die Kinder in ihrem musikalischen Lernen zu unterstützen, brauchen die Lehrpersonen neben ihrem fachlichen Können und Wissen Offenheit, Sensibilität und Wissen gegenüber dem, was bei den Kindern in ihren musikalischen Aktionen und Interaktionen gerade jetzt geschieht. Was können Kinder schon und was bringen sie mit? Wie können sie dies im Alltag von Kindergarten und Schule zeigen? Was kann in den musikalischen und musikbezogenen Handlungen beobachtet werden und wie können die Kinder für die nächsten Schritte unterstützt werden (vgl. Küntzel 2009)? „Die achtsame Wahrnehmung der Aktivitäten der Kinder und ihrer musikalischen Erfahrungswelten [rückt] an den Anfang.

2 Zur Begrifflichkeit der Fachwissenschaft Abschnitt 6.1. 
Musikalisches Experimentieren und Gestalten kann so unmittelbar aus dem Tun der Kinder entstehen" (Beck-Neckermann 2014, 6). Dabei braucht es Wissen und Erfahrungen, wie Impulse der Kinder aufgenommen und in einen nächsten Lernschritt weitergeführt werden können.

In Bezug auf die in Abschnitt 2 erwähnten Bestimmungen von Musik gibt es unterschiedliche Perspektiven, Zielsetzungen und Handlungsfelder im Lehren von Musik, die hier zur Verdeutlichung getrennt dargestellt werden. Im realen Musikunterricht spielen sie zusammen, vermischen und durchdringen sich. Musikunterricht, welcher sich am Werk orientiert, hat vereinfachend gesprochen einerseits zum Ziel, Musik so gut wie möglich mit den Kindern zu gestalten und es ihnen zu ermöglichen, tradierte Musikwerke aus verschiedenen Zeiten aktiv kennenzulernen und zum Klingen zu bringen. Werke entstehen andererseits neu durch Kompositionen der Kinder. Ausgehend von klingendem Material und Strukturierungsimpulsen werden Musikstücke erfunden und festgelegt. ${ }^{3}$ Das Erlernen, Üben und Aufführen von tradiertem stufenspezifischem Liederrepertoire und die Auseinandersetzung mit tradierter Musik durch Hören, Interpretieren und Verstehen stehen im Fokus.

Unterricht, welcher die gestaltende, orientierende und gelassene Lebensführung thematisiert, führt zur Bildung im Sinne von ästhetischer, psychischer, logischer, ethischer, sozial-psychosomatischer und pragmatischer Stimmigkeit. Das wird ermöglicht durch die Förderungen der musikalischen Selbsttätigkeit, durch die Auseinandersetzung mit musikalischen Grunderfahrungen, durch die Ausdifferenzierung von Fühl-, Denk- und Verhaltensmustern, durch Begegnungen mit kulturellem Material in den Handlungsfeldern "Singen“, „Instrumentalspiel“, „Bewegung und Tanzen“, „Wahrnehmen und Erleben“ und „Denken und Symbolisieren" und durch das Verbinden von Musik mit verschiedenen Gestaltungsformen (vgl. Dartsch 2010, 321-323). ${ }^{4}$

Steht der Erlebnischarakter von Musik im Vordergrund, liegt der Schwerpunkt auf der Ermöglichung von ästhetischen Erfahrungen. ${ }^{5}$ Grob gesagt geht es dabei um die fortschreitende Differenzierung von akustischen und leiblichen Wahrnehmungen und um die Kreation, Entwicklung und Ausarbeitung von musikalischen Gestaltungsformen. Musik bedient sich einer nonverbalen klanglichen, analogen und metaphorischen Sprache. Ästhetische Erkenntnis, oder im engeren Sinne

3 In Janet Mills in dritter Auflage vorliegendem Buch „Music in the Primary School“ steht im Vorwort: „I think of music education as an active experience in which pupils compose, perform, and listen“ (Mills 2009). Interessant ist hier die Reihenfolge: „compose, perform and listen“. Komponieren steht hier am Anfang der musikalischen Ausbildung.

4 Der schweizerische Lehrplan formuliert sechs Kompetenzbereiche: MU.1 Singen und Sprechen, MU.2 Hören und Sich-Orientieren, MU.3 Bewegung und Tanzen MU.4 Musizieren, MU.5 Gestaltungsprozesse und MU.6 Praxis des musikalischen Wissens (D-EDK 2016, 6-7).

5 Zur kritischen Auseinandersetzung mit dem Begriff vgl. z.B. Mattenklott (2004). 
Erkenntnis im musikalischen Bereich, „macht uns die Welt zugänglich und verständlich, indem sie Ähnlichkeiten aufspürt und ein Netz an Beziehungen knüpft, das uns die Abgründe des Nicht-Verstehens vergessen lässt" (Brandstätter 2008, 37). Eng mit diesem Ansatz verbunden sind Bezüge zu anderen Ausdrucks- und Gestaltungsformen wie Tanz, bildenden Künsten und Sprache. Dieser Unterricht ist darauf ausgerichtet, musikalische Erfahrungs- und Gestaltungsräumen für die Kinder zu schaffen, sie in ihrer schöpferischen Arbeit zu unterstützen und zu begleiten und mit den Kindern ihre musikalischen Prozesse und Produkte zu reflektieren (vgl. Meyer, 2004).

Wird Musik als Handlung verstanden, orientiert sich der Unterricht an musikalischen und musikbezogenen Aktivitäten, welche ,ästhetisches Verstehen vertief[en] und erkennendes Verstehen förder[n]" (Schatt 2007, 117). Im Handbuch für Musikpädagogik werden als „didaktische Handlungsfelder“ die folgenden aufgeführt: Singen, Instrumentalspiel, Wahrnehmen und Hören, Improvisation, Komposition, Tanzen/Bewegen, Musik und andere künstlerische Ausdrucksformen, Reflexion (Dartsch et al. 2018, 7). Der Fokus der musikpädagogischen Arbeit aus diesem Ansatz von Musikverständnis liegt auf der Verfeinerung und der Ausdifferenzierung der Art und Weise, wie gesungen, gespielt oder getanzt wird, der Prozess ist wichtig. Dabei sind zu erreichende Wahrnehmungs-, Erfahrungsund Handlungskompetenzen und das damit verbundene Reflektieren, Benennen und Verstehen leitend, damit Musikunterricht nicht zu einem reinen aktionistischen Musikmachen wird (Schatt 2007, 74).

Geht es um das Verständnis von Musik als Bildungsinhalt „[... ] leistet der Musikunterricht einen wichtigen Beitrag an die kulturelle Bildung, indem er allen Kindern und Jugendlichen das Bilden und Schärfen eines ästhetischen Urteils und den aktiven Umgang mit Musik ermöglicht. Übergeordnetes Ziel ist eine musikalische Grundbildung, ausgerichtet auf Förderung von Kreativität, performative Fertigkeiten und ästhetischen Sinn sowie auf die Vermittlung von Kenntnissen in Kunst und Kultur" (D-EDK 2016, 2). Damit geht es um elementare Erfahrungen und damit verbundene Kenntnisse in einem bestimmten kulturellen Kontext, welche aktiv gelernt, angewendet, gezeigt und beurteilt werden.

Die Thematisierung von Stimmungen und Atmosphären durch ihre klanglichen Realsierungen oder durch Vertonungen von Bildern und Szenen steht bei der Auffassung von Musik als Trägerin von Bedeutungen und Wirkungen im Vordergrund. Leitend dabei sind bewusste Analogiebeziehungen zwischen Wirkungen von Musik und Wirkungen von Bildern, Bewegungen, sprachlichen Äusserungen und Stimmungen (Brandstätter 2009). In diesen Bereich gehört auch der Einsatz von Musik als Signal in Alltagsritualen, beispielsweise das Erklingen von Klängen zur Strukturierung des Unterrichts und zur Beruhigung oder Trommeln, um die hohen Energien in einer Gruppe konstruktiv zu koordinieren. Musik wird als Symbol, als Zeichen, als Mittel für etwas anderes genutzt. 
Musik als Möglichkeit der Selbstdefinition ist mit der Entwicklung und der Modifizierung des musikbezogenen Selbstkonzepts korreliert: „Ein Selbstkonzept ist das, was ein Mensch über sich selbst denkt: Was er ist und was er kann" (Spychiger 2013, 18). In Kindergarten und Schule sollen Gelegenheiten geschaffen werden, bei denen die Kinder zeigen können, was sie können. Die Kinder bewegen sich spontan zu Musik, die sie hören, sie singen einfach so für sich und mit anderen und können passende Lieder finden. Sie spielen gern auf und mit Instrumenten und erfinden Klangabläufe, sie können zu Musik Geschichten erfinden, sie können gebannt Musik zuhören und vieles mehr (vgl. Küntzel 2009). Das Zeigen, das Gehörtwerden und die erhaltenen Rückmeldungen von anderen Kindern und der Lehrkräfte stützen ein positives musikbezogenes weitgefasstes Selbstkonzept. Es ist aus diesen Ausführungen nachvollziehbar, dass es nicht ein einziges verbindliches musikpädagogisches Konzept gibt, welches alle diese Aspekte abdecken kann. So liegen für die musikalische Ausbildung für den 1. Zyklus mehrere unterschiedliche Modelle und Konzeptionen vor, die mit unterschiedlicher Gewichtung auf diesen Orientierungen und auf verschiedenen Menschenbildern und Auffassungen von Musik basieren (vgl. Bailer 2017; Lehmann-Wermser 2016; Schatt 2007). In den letzten Jahren haben sich zwei im deutschsprachigen Raum massgebende Konzepte, die Elementare Musikpädagogik und der Aufbauende Musikunterricht, angenähert und ergänzen sich gegenseitig. Die Elementare Musikpädagogik geht stark vom Blick auf das Kind aus, orientiert sich am Spiel und am Experiment sowie am Einbezug des Körpers. Der Aufbauende Musikunterricht kommt eher von der Sekundarstufe her, geht stärker von der Sache ,Musik aus und orientiert sich an einem systematischen Aufbau (Oravec/Weber-Krüger 2016, 21). Wenige aktuelle Lehrmittel beziehen sich mehr oder weniger explizit auf diese Modelle und Menschenbilder (Beck-Neckermann 2014; Fuchs 2010; Funk/Nykrin 2008). Die Mehrzahl orientiert sich an den in den Lehrplänen formulierten Kompetenzbereichen (Held/Lang/Steffen 2010; Jakobi-Murer/ Rohrbach/Leupold/von Wurmb/Schmidmeier 2017; Merki/Berger 2015) und noch häufiger sind Sammlungen von rezeptartigen Unterrichtsideen und Stundenbildern (Grohé/Jasper 2016; Grohé/Junge 2014; Grohé/Junge/Müller 2010; Joschko/2013a/2013b; Mayer-Makein/Bauder-Reissing/Tille-Koch 2011; Silberg 1999) oder Liederbücher mit methodischen Hinweisen anzutreffen (Bosshart/ Bosshart 2006; Heeb/Schär 2011; Zihlmann/Zihlmann 2017).

Um einen Überblick über die unterschiedlichen Ansätze zum Musikunterricht zu erhalten, wurde basierend auf der Auseinandersetzung mit der Philosophy of music education ${ }^{6}$ aus dem englischsprachigen Raum (Jorgensen 2011; Schippers 2010) ein Rahmenmodell (vgl. Abb. 1) entwickelt, welches die unterschiedlichen Ansätze in sechs Dimensionen (Musik, Lehren, Lernen, Inhalte, Bewertungen

6 Zur Diskussion zum Begriff vgl. Kertz-Welzel (2014). 
und Interaktionen) verortet und die Spanungsfelder zwischen verschiedenen Konzeptionen darstellt. Vereinfachend und stark reduzierend werden die Begriffe „Konservatorium“ und „Laboratorium“ als metaphorische Überbegriffe für die beiden Seiten des Modells verwendet. ${ }^{7}$ Es wird deutlich, dass unterschiedlichen Formen von Musikunterricht andere Schwerpunkte setzen und es nicht eine einzig richtige Form geben kann. Musikunterricht wird als dynamisches Geschehen verstanden, welches sich auf den Achsen der Dimensionen des Rahmens bewegt und einen Ausgleich zwischen den Polaritäten anstrebt (Zurmühle 2021).

\begin{tabular}{|c|c|c|}
\hline Bestehende Musik & & Entstehende Musik \\
\hline Notiert & & Nicht notiert \\
\hline Strukturierter Aufbau & Lehren & Spontan, situativ \\
\hline Anleitungen & & «Wildes Lernen» \\
\hline Rezeptives Lernen & & $\begin{array}{c}\text { Konstruktivistisches } \\
\text { Lernen }\end{array}$ \\
\hline Sache im Zentrum & Inhalt & Mensch im Zentrum \\
\hline Produkt & Bewertungen & Prozess \\
\hline $\begin{array}{l}\text { Hierarchische } \\
\text { Interaktion }\end{array}$ & Interaktionen & $\begin{array}{l}\text { Dialogische } \\
\text { Interaktion }\end{array}$ \\
\hline Konservatorium & & Laboratorium \\
\hline
\end{tabular}

Abb. 1: Rahmenmodell

\section{Musik im Rahmen der Ausbildung zur Generalistin bzw. zum Generalisten}

Das Studium im Fachbereich „Musikpädagogik“ verfolgt grundsätzlich zwei Schwerpunkte: 1. die fachwissenschaftliche Ausbildung und 2. die fachdidaktische

7 Damit werden keine realen Orte gemeint; Dewey verwendet „Museum“ und „Laboratorium“ (vgl. Allsup 2016, 70). 
Ausbildung der Studierenden. ${ }^{8}$ Die beiden Bereiche greifen ineinander, da ohne ein fachpraktisches musikalisches Können keine musikbezogene Unterrichtsgestaltung für die Kinder durchgeführt werden kann. Die Inhalte und Lernformate in der musikalischen Ausbildung orientieren sich am vorgestellten Rahmenmodell und thematisieren vorhandene wie entstehende Musik, strukturierte und offene Lehr- und Lernformen und produkt- und projektorientierte Rückmeldungen und Beurteilungen.

Die Konzeption des Studienaufbaus versucht auf unterschiedliche Herausforderungen konstruktiv zu reagieren:

1. Die Heterogenität der Studierenden: Diese kommen mit sehr unterschiedlichen musikalischen Voraussetzungen. Von professionellen Musikerinnen und Musikern bis zu Studierenden, die seit längerer Zeit keine musikalischen Erfahrungen mehr gemacht haben. Darauf wird mit dem individuellen Instrumentalunterricht, einem freiwilligen Tutoringangebot und individualisierten Leistungsnachweisen reagiert.

2. Die kurze Dauer der Ausbildung: Die Entwicklung und die Festigung von musikalischen handlungsorientierten und gruppenorientierten Kompetenzen brauchen Zeit zur gemeinsamen Erfahrung und Übung. Die Seminare sind vorwiegend handlungsorientiert gestaltet und der Erwerb von Wissen durch Fachliteratur wird in die Selbststudienzeit verlegt.

3. Die Verschiedenheit von musikdidaktischen Konzeptionen: Darauf wird reagiert, indem im Grundstudium mindestens zwei unterschiedliche Konzeptionen vorgestellt und in einem übergeordneten Rahmenmodell verortet werden. Der Studienaufbau folgt einer Entwicklung von geführten und strukturierten zu offeneren und freieren Lern- und Aktionsformen.

\subsection{Die fachwissenschaftliche Ausbildung}

Die fachwissenschaftliche Ausbildung der Studierenden beinhaltet einerseits Einzelunterricht in einem Instrument oder in Gesang. ${ }^{9}$ Der hoch individualisierte Unterricht knüpft an den musikalisch sehr heterogenen Vorerfahrungen der Stu-

8 Die Begriffe „Fachwissenschaft“ und „Fachdidaktik“ erfordern eine kurze Klärung. Das Verständnis des Begriffs „Fachdidaktik Musik“ ist relativ klar beschreibbar; es geht um die wissenschaftsbasierte Auseinandersetzung mit den Grundbedingungen des Lernens und Lehrens von Musik in den Institutionen Kindergarten und Primarschule. Der Begriff „Fachwissenschaft Musik“ wird ausserhalb der Schweiz kaum verwendet und ist nicht mit ähnlichen Begriffen wie „Musikwissenschaft“ oder „wissenschaftliche Musikpädagogik“ gleichzusetzen. In unserem Verständnis handelt es sich im Wesentlichen um ein fachpraktisches Ausbildungselement, welches es den Studierenden ermöglicht, ihre eigenen musikalischen Fähigkeiten und Kompetenzen in den verschiedenen Bereichen zu vertiefen und zu reflektieren. Der Begriff „Fachwissenschaft Musik“ wird im Folgenden in dieser Bedeutung und im Sinne der Konsistenz mit den anderen Fachbereichen verwendet.

9 Eine Lektion pro Woche über ein Jahr. 
dierenden an und fördert die Studierenden in der Anwendung des Instruments und der eigenen Singstimme. Die instrumenten- und gesangsspezifischen Themen werden mit musiktheoretischen und schulpraktisch relevanten Themen verbunden (Entwicklung der eigenen Singstimme, Stabilisierung der rhythmischen Sicherheit, Praxis der Solmisation mit dem Instrument, Grundlagen der Musiktheorie, Improvisieren, Komponieren, Notation). Die Studierenden komponieren ein eigenes Lied, machen eine Theorieprüfung und begleiten sich gegenseitig in stufenbezogenen Liedern. Das gemeinsame Musizieren mit der Stimme, Instrumenten und dem eigenen Körper steht im Fokus von seminaristischen Gruppenveranstaltungen. Die Studierenden können sich in verschiedenen Themenbereichen vertiefen, die sich aus den Kompetenzbereichen des Lehrplans 21 ableiten: 1. Singen und Stimme, 2. Hörsensibilisierung und -differenzierung, 3. Bewegung und Rhythmus und 4. Improvisieren, komponieren und arrangieren. Die individuelle Arbeitsleistung ist eine musikalische Performance in Viergruppen.

\subsection{Die fachdidaktische Ausbildung}

In der fachdidaktischen Ausbildung werden die Grundlagen und Grundprinzipien für musikalisches und musikbezogenes Lernen der Kinder vermittelt. Im Zentrum steht in Abgrenzung zu einem individualisierten und spezialisierten Musikunterricht an den Musikschulen das grundlegende und vorwiegend in Gruppen stattfindende gemeinsame aktiv handelnde Musiklernen. Ein weiterer wesentlicher Teil für den Musikunterricht im 1. Zyklus sind musikalische Unterrichtsgestaltungsformen wie Rituale, Signale und Kommunikationsformen. Die Studierenden werden in mindestens zwei unterschiedliche Unterrichtskonzeptionen eingeführt, die Elementare Musikpädagogik und den Aufbauenden Musikunterricht, und lernen darauf beruhende Lehrmittel kennen. Diese Kenntnisse werden praktisch in der Gruppe umgesetzt und im Leistungsnachweis überprüft. Hier zeigt sich deutlich die Verbindung zwischen dem fachwissenschaftlichen Können und dem fachdidaktischen Kennen. In der Weiterführung stehen analog zur Fachwissenschaft vier Themenbereiche zur Wahl: 1. Musikalische Spiele und Rituale, 2. Freiheit und Struktur im Musikunterricht, 3. Musikalische Projekte mit Kindern und 4. Themen musikalisch gestalten. Die individuelle Arbeitsleistung besteht in der Verfassung eines Tutorials für Kinder und einer theoriegestützten Diskussion eines Videos zu einer musikbezogenen Unterrichtssituation. Mit einer Wahl des Schwerpunkts „Musik“ können die Studierenden ihre Fähigkeiten in anderen Themenbereichen verbreitern und durch die individuellen Arbeitsleistungen (Planung, Begründung, Durchführung und Evaluation von musikalischen Produkten oder Projekten mit Kindern oder für Kinder) vertiefen. 


\section{Herausforderungen und Chancen der musikalischen Ausbildung im Rahmen der Ausbildung zur Generalistin bzw. zum Generalisten}

Wie bei anderen fachlichen Ausbildungen sind die oben erwähnten Themen ,Heterogenität bei den musikalischen Voraussetzungen der Studierenden' und ,zu wenig Zeit für die Bildung und die Vertiefung von fachlichem Können' die grössten Herausforderungen. Die Heterogenität bei den Voraussetzungen der Studierenden hat mehrere Gründe. Die musikalische Ausbildung kann auf der einen Seite seit der frühen Kindheit kontinuierlich und durch privaten Musikunterricht erfolgt sein. Auf der anderen Seite kann sie sehr bescheiden sein, wenn Musik in der Sekundarstufe II nicht mehr gewählt wurde und kein formaler Musikunterricht mehr stattgefunden hat. Das kann auch verbunden sein mit einem sehr negativ behafteten musikbezogenen Selbstkonzept, zu dessen Umdeutung viel bewusste erfahrungsbasierte Reflexion notwendig ist (vgl. Spychiger 2013). Die geringe Zeit für die fachliche Ausbildung wird deutlich sichtbar, wenn zum Vergleich fachlich orientierte Ausbildungen herangezogen werden, die auf die gleiche Altersgruppe ausgerichtet sind. So ist die Ausbildung in "Musik und Bewegung" ein komplettes Bachelorstudium, welches mit einem Masterstudium ergänzt werden kann. („Bachlor Musik und Bewegung “10). Zusätzlich grenzen die engen strukturellen Vorgaben wie die vorgegebenen Zeitgefässe für den Unterricht, die unbedingt notwendigen und möglichst rekurssicheren Überprüfungen von Leistungen in jedem Modul, die Notwendigkeit der Kreditierung und die finanziellen Ressourcen die Möglichkeiten ein, um grössere musikalische Projekte mit einem musikalisch hohen Anspruch, mit dem Einbezug von schwierig beurteilbaren kreativen Anteilen und damit mit einem fachlich hohen Lerngewinn zu realisieren oder den Studierenden zusätzliche Formen der musikalischen Praxis zu ermöglichen.

Als Chance ermöglicht die musikalische Ausbildung den Studierenden den Erwerb von Kenntnissen und Erfahrungen in einem ästhetisch-musischen Fachbereich, welcher in der Lebenswelt der Kinder eine sehr grosse Rolle spielt (Badur 2014). Dies erfolgt mehrheitlich in musikalischen Handlungen und Praktiken in Gruppen, welche von den Studierenden sehr geschätzt werden. Musik als ästhetische Erfahrung ${ }^{11}$ rückt „das Subjekt und eine ihm eigene spezifische ästhetische Einstellung und Haltung in den Mittelpunkt der Aufmerksamkeit [...]“ (Mat-

$10 \mathrm{Vgl}$. die Informationen zu diesem Studiengang unter: https:/www.fhnw.ch/de/studium/musik/ klassik/bachelor-musik-und-bewegung.

11 Zum Begriff: „Angesichts der Fülle unterschiedlicher Erfahrungen, die unter dem Begriff der ästhetischen Erfahrung subsumiert werden können, ist eine allgemeine und abschließende Definition nicht leistbar und auch nicht unbedingt wünschenswert - sie würde dem offenen Charakter des Ästhetischen widersprechen" (Brandstätter, 2017). 
tenklott 2004, 12). Aktiv und verstehend begegnen die Studierenden der Musik als Teil von verschiedenen individuellen, kulturellen, sozialen und historischen Lebenswelten, die sie mitgestalten können.

Durch die fachliche Ausbildung in Musik wird auch die Gestaltung von Unterricht und schulischem Alltag mit Musik ermöglicht. Musik ist in Kindergarten und Primarschule täglich integriert in gemeinsamen Ritualen, Übergängen und Momenten des gemeinsamen Musizierens. Grosse Chancen bestehen in der Einbindung des Fachbereichs ,Musik' in interdisziplinäre Projekte und Lehrveranstaltungen, beispielsweise in Seminare zu Musik und Bild, zu Musik und informatischer Bildung oder zu Musik und Bewegung: „Musiklernen ist Aneignung von Wirklichkeit mit musikalischen Mitteln“ (Küntzel 2009, 3).

\section{Literatur}

Allsup, R. E. (2016). Remixing the classroom: toward an open philosophy of music education. Bloomington: Indiana University Press.

Altenmüller, E. (2018): Vom Neandertal in die Philharmonie. Warum der Mensch ohne Musik nicht leben kann. Berlin: Springer.

Badur, I.-M. (2014): Musikbezogene Aktivitäten von Kindern im Grundschulalter. Inaugural-Dissertation. Giessen: Justus-Liebig-Universität.

Bailer, N. (2017): Insel-Bilder: musikdidaktische Konzeptionen im Diskurs. Rum-Esslingen: Helbling.

Beck-Neckermann, J. (2014): Mit Kindern Musik entdecken. Musikalisches Experimentieren und Gestalten in der frühpädagogischen Bildungsarbeit. Gütersloh: Bertelsmann Stiftung.

Böhme, G. (2001): Aisthetik Vorlesungen über Ästhetik als allgemeine Wahrnehmungslehre. München: Fink.

Bosshart, E. \& Bosshart, E. (2006): Eifach singe. Das Kinderliederbuch für die Vorschul- und Grundstufe. Zürich: Lehrmittelverlag des Kantons Zürich.

Brandstätter, U. (2008): Grundfragen der Ästhetik: Bild, Musik, Sprache, Körper. Köln: Böhlau.

Brandstätter, U. (2009): Bildende Kunst und Musik im Dialog - Ästhetische, zeichentheoretische und wahrnehmungspsychologische Überlegungen zu einem kunstspartenübergreifenden Konzept ästhetischer Bildung. Augsburg: Wissner.

Brandstätter, U. (2013): Erkenntnis durch Kunst: Theorie und Praxis der ästhetischen Transformation. Köln: Böhlau.

Brandstätter, U. (2017): Ästhetische Erfahrung. In: Kulturelle Bildung online. Online unter: https:// www.kubi-online.de/artikel/aesthetische-erfahrung.

D-EDK (2016): Lehrplan 21: Musik. Luzern: D-EDK.

Dartsch, M. (2010): Mensch, Musik und Bildung. Grundlagen einer Didaktik der Musikalischen Früherziehung. Wiesbaden: Breitkopf und Härtel.

Dartsch, M. (2014): Musik lernen - Musik unterrichten. Eine Einführung in die Musikpädagogik. Wiesbaden: Breitkopf und Härtel.

Dartsch, M., Knigge, J., Niessen, A., Platz, F. \& Stöger, C. (2018): Handbuch Musikpädagogik Grundlagen - Forschung - Diskurse. Münster: Waxmann.

Evelein, F. (2015): Kooperative Lernmethoden im Musikunterricht. 188 Partner- und Gruppenaktivitäten für die Klassen 5 bis 12. Innsbruck: Helbling.

Fuchs, M. (2010): Musik in der Grundschule neu denken - neu gestalten: Theorie und Praxis eines aufbauenden Musikunterrichts. Rum/Innsbruck: Helbling. 
Funk, J. \& Nykrin, R. (2008): Musik und Tanz für Kinder. Unterrichtswerk zur Früherziehung. Mainz: Schott.

Gembris, H. (2013): Grundlagen musikalischer Begabung und Entwicklung. Augsburg: Wissner.

Geuen, H. (2018): Musikbegriffe. In: M. Dartsch, J. Knigge, A. Niessen, F. Platz \& C. Stöger, (2018), Handbuch der Musikpädagogik. Münster: Waxmann, 18-24.

Grohé, M. \& Jasper, C. (2016): Methodenrepertoire Musikunterricht. Zugänge - Lernwege - Aufgaben. Innsbruck: Helbling.

Grohé, M. \& Junge, W. (2014): Musikspiele 2. 77 Spiele rund um den Musikunterricht. Innsbruck: Helbling.

Grohé, M., Junge, W. \& Müller, K. (2010): Musikspiele. 99 Spiele rund um den Musikunterricht. Rum/Innsbruck: Helbling.

Gruhn, W. (2003): Kinder brauchen Musik: Musikalität bei kleinen Kindern entfalten und fördern. Weinheim: Beltz.

Gruhn, W. (2010): Anfänge des Musiklernens: eine lerntheoretische und entwicklungspsychologische Einführung. Hildesheim: Olms.

Gruhn, W. (2014): Der musikalische Mensch. Evolution, Biologie und Pädagogik musikalischer Begabung. Hildesheim: Olms.

Gruhn, W. (2017): Was der Körper nicht lernt, lernt der Kopf nimmermehr ... Lerntheoretische Überlegungen zur Bedeutung der Leiblichkeit des Lernens. In: L. Oberhaus \& C. Stange (Hrsg.), Musik und Körper. Bielefeld: transcript, 105-119

Gruhn, W. \& Röbke,P. (2018): Musiklernen Bedingungen - Handlungsfelder - Positionen. Innsbruck: Helbling.

Hammel, L. (2011): Selbstkonzepte fachfremd unterrichtender Musiklehrerinnen und Musiklehrer an Grundschulen. Eine Grounded-Theory-Studie. Berlin: LIT.

Heeb, R. \& Schär, H. (2011): Sing mit! Schweizer Singbuch. Unterstufe. Rorschach: Kantonaler Lehrmittelverlag St. Gallen.

Held, R., Lang \& Steffen, C. (2010): Kreschendo. 1/2 Musik für die 1. und 2. Klasse. Zug: Comenius.

Jakobi-Murer, S., Rohrbach, K., Leupold, R., von Wurmb, I. D. \& Schmidmeier, C. (2017): Tipolino 1: fit in Musik für den Unterricht an allgemeinbildenden Schulen und Kindergärten, Zyklus 1 (Kindergarten bis 2. Schuljahr). Innsbruck: Helbling.

Jorgensen, E. R. (2011): Pictures of music education. Counterpoints: music and education. Bloomington: Indiana University Press.

Joschko, J. (2013a): Kompetenzorientierter Musikunterricht 1. Klasse. Donauwörth: Auer.

Joschko, J. (2013b): Kompetenzorientierter Musikunterricht 2. Klasse. Donauwörth: Auer.

Kertz-Welzel, A. (2014): Musikpädagogische Grundbegriffe und die Internationalisierung der Musikpädagogik. Ein unlösbares Dilemma? In: J. Vogt, F. Hess \& M. Brenk (Hrsg.), (Grund-)Begriffe musikpädagogischen Nachdenkens. Berlin: LIT, 19-35.

Khittl, C. (2017): Musikpädagogik: Vom „Anything Goes“ über disziplin-loses Durcheinander zur (anthropischen) Trans-Disziplin. In: N. Bailer \& H. Enser (Hrsg.), Insel-Bilder. Musikdidaktische Konzeptionen im Diskurs. Innsbruck: Helbling, 9-28.

Küntzel, B. (2009): Musikunterricht. Baltmannsweiler: Schneider Verlag Hohengehren.

Lehmann-Wermser, A. (2016): Musikdidaktische Konzeptionen. Ein Studienbuch. Augsburg: Wissner.

Mattenklott, G. (2004): Ästhetische Erfahrung in der Kindheit: theoretische Grundlagen und empirische Forschung. Weinheim: Juventa.

Mayer-Makein, M., Bauder-Reissing, U. \& Tille-Koch, J. (2011). Fachfremd Musik unterrichten. Ideen und Methoden für motivierenden und erfolgreichen Musikunterricht Grundschule. Kerpen-Buir: Kohl. 
Merki, B. \& Berger, E. (2015): MusAik 1. Musiklehrmittel Kindergarten und 1.-3. Schuljahr. Rorschach: Lehrmittelverlag St. Gallen.

Meyer, C. (2004): Inszenierung musikalisch-ästhetischer Erfahrungsräume in der Elementaren Musikpädagogik. In: J. Ribke \& M. Dartsch (Hrsg.) Gestaltungsprozesse erfahren - lernen - lehren. Regensburg: Con Brio, 93-99.

Mills, J. (2009): Music in the primary school. Oxford: Oxford University Press.

Oravec, L. \& Weber-Krüger, A. (2016): Musiklernen in der Grundschule. Impulse aus elementarer und schulischer Musikpädagogik. Essen: Die blaue Eule.

Ott, T. (2018): Konzeptionen und zentrale Orientierungen für schulischen Musikunterricht. In: M. Dartsch, J. Knigge, A. Niessen, F. Platz \& C. Stöger (Hrsg.), Handbuch Musikpädagogik Grundlagen - Forschung - Diskurse. Münster: Waxmann, 284-288.

Podbregar, N. (2013): Weichen fürs Sprechenlernen werden im Mutterleib gestellt. In: wissenschaft. de. Online unter:

https://www.wissenschaft.de/umwelt-natur/weichen-fuers-sprechenlernen-werden-immutterleib-gestellt/.

Richter, C. (2019): Musik - ein Lebensmittel. Überlegungen zu einer Anthropologie der Musik. Augsburg: Wissner.

Schatt, W. (2007): Einführung in die Musikpädagogik. Darmstadt: Wissenschaftliche Buchgesellschaft.

Schellberg, G. (2016): „Mein musikalisches Selbstbewusstsein ist gewachsen!“ - Wirkungen eines Pflichtseminars für Grundschullehrpersonen. In: L. Oravec \& A. Weber-Krüger (2016), Musiklernen in der Grundschule. Impulse aus elementarer und schulischer Musikpädagogik. Essen: Die blaue Eule, 143-171.

Schippers, H. (2010): Facing the music: shaping music education from a global perspective. Oxford: Oxford University Press.

Silberg, J. (1999): Aber ich kann doch gar nicht singen! Musik unterrichten für „Unmusikalische“. Mülheim an der Ruhr: Verlag an der Ruhr.

Spychiger, M. (2013): Das musikalische Selbstkonzept. In: Üben \& Musizieren, Zeitschrift für Instrumentalpädagogik und musikalisches Lernen, 30. Jg. (H. 6), 18-21.

Stadler Elmer, S. (2014): Bildungsakzent. Musik für kleine Kinder. In M. Dartsch, M. (Hrsg.), Musik im Vorschulalter. Dokumentation Arbeitstagung 2013. Kasel: Bosse, 58-67.

Stadler Elmer, S. (2015): Kind und Musik: Das Entwicklungspotenzial erkennen und verstehen. Berlin: Springer.

Weber-Krüger, A. (2014): Bedeutungszuweisungen in der Musikalischen Früherziehung. Integration der kindlichen Perspektive in musikalische Bildungsprozesse. Münster: Waxmann.

Zihlmann, E. \& Zihlmann, K. (2017): Hoppelihopp: 20 Lieder für Kinder von 4-8 Jahren. Basel: Zytglogge.

Zurmühle, J. (2021): Entweder-oder, sowohl-als-auch, weder-noch, gar-nichts: Rahmenmodell für Unterrichtskonzepte für den schulischen Musikunterricht in Kindergarten und Primarschule. In: J. Huber, M. A. Camp, O. Blanchard, S. Chatelain, F. Joliat, R. Steiner \& J. Zurmühle (Hrsg.), Kulturen der Schulmusik. Zürich: Chronos. 